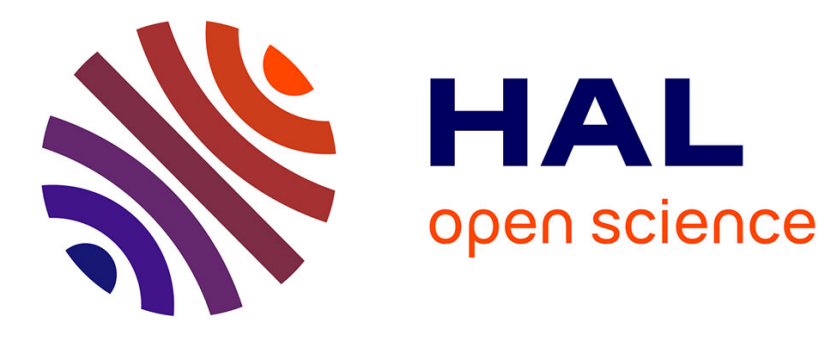

\title{
IP Mobility in Aeronautical Communications
}

\author{
Alexandre Tran Nguyen, Alain Pirovano, Nicolas Larrieu, Alain Brossard, \\ Stéphane Pelleschi
}

\section{To cite this version:}

Alexandre Tran Nguyen, Alain Pirovano, Nicolas Larrieu, Alain Brossard, Stéphane Pelleschi. IP Mobility in Aeronautical Communications. Nets4, 13th International Workshop on Communication Technologies for Vehicles, Nets4Aircraft and UAV session, May 2018, Madrid, Spain. pp 16-26 / ISBN 978-3-319-90370-5, 10.1007/978-3-319-90371-2_2 . hal-01785047

\section{HAL Id: hal-01785047 https://hal-enac.archives-ouvertes.fr/hal-01785047}

Submitted on 4 May 2018

HAL is a multi-disciplinary open access archive for the deposit and dissemination of scientific research documents, whether they are published or not. The documents may come from teaching and research institutions in France or abroad, or from public or private research centers.
L'archive ouverte pluridisciplinaire HAL, est destinée au dépôt et à la diffusion de documents scientifiques de niveau recherche, publiés ou non, émanant des établissements d'enseignement et de recherche français ou étrangers, des laboratoires publics ou privés. 


\title{
IP Mobility in Aeronautical Communications
}

\author{
Alexandre Tran ${ }^{1,2}$, Alain Pirovano ${ }^{1}$, Nicolas Larrieu ${ }^{1}$, \\ Alain Brossard ${ }^{2}$, Stéphane Pelleschi ${ }^{2}$ \\ ${ }^{1}$ ENAC, TELECOM/ReSCo, Toulouse, France \\ \{Alexandre.Tran, Alain.Pirovano, \\ Nicolas.Larrieu\} @recherche.enac.fr \\ 2 Rockwell Collins \\ \{Alain.Brossard, Stephane.Pelleschi\}@rockwellcollins.com
}

\begin{abstract}
In the sake of modernization, aviation stakeholders decided that the future aviation network infrastructure, in particular for air-ground communication systems, will move towards IP based networks. It has been referred to in the International Civil Aviation Organization as Aeronautical Telecommunication Network/Internet Protocol Suite. Due to the heterogeneous communication environment, it is necessary to support handover between different access technologies and access networks. In this article, we first define the very specific aeronautical communication environment. Our main contribution is a performance assessment of the most deployed network protocols capable of managing IP mobility within the aeronautical environment. We focus our analysis on the Mobile IPv6 protocol and implementation issues of a representative aeronautical network in Omnet++.
\end{abstract}

Keywords: IP mobility, air ground communications, MIPv6.

\section{Introduction}

The current aeronautical communication infrastructure for Air Traffic Management (ATM) has to evolve in order to respond to an endless increase in air traffic and to support more stringent data link communications. In this way, aviation stakeholders gathered in several groups such as International Civil Aviation Organization (ICAO), European Organization for Civil Aviation Equipment (EUROCAE), Radio Technical Commission for Aeronautics (RTCA) and Airlines Electronic Engineering Committee (AEEC), are working on a future IP-based Aeronautical Telecommunication Network (ATN/IP). Its goal is to interconnect all the aeronautical subnetworks with the IPv6 protocol.

The aeronautical environment is different from other communication domains (e.g personal wireless communication in $4 \mathrm{G}$ networks or vehicular communications). The two main key problems are the global mobility and the safety-related data which have very stringent quality of services $(\mathrm{QoS})$ requirements. Whereas some solutions have already been proposed in [1], no solution has been taken on yet by the aviation industry. 
To handle the node mobility in IPv6, the Internet Engineering Task Force (IETF) has developed Mobile IPv6 (MIPv6) [2]. It allows a seamless communication between a mobile node and its correspondent node via a Home Agent (HA). In this article, we investigate MIPv6 for an aeronautical scenario through the xMIPv6 model of the INET framework [3].

The remainder of the paper is organized as follows: Section 2 provides an overview of the aeronautical communications environment and its evolution towards IP based networks. Section 3 highlights the need of managing the node mobility under different IP networks and presents some dedicated protocols, with their strengths and flaws. In section 4, we explain our simulation model for MIPv6 in a context of an aeronautical environment. We then analyze the first results and conclude with several suggestions for our simulation model and the IP mobility management in an aeronautical environment.

\section{$2 \quad$ Aeronautical Air Ground Communications and Data link}

\subsection{From analog voice to Data link}

The first radio transmitter has been invented and tested by AT\&T in 1917, allowing for the first time voice communications between ground personnel and pilots. But, it was only in 1935 that airborne radios were considered reliable and efficient enough to be widely deployed on existing aircraft. These air-ground communication means were proposed in order to increase air safety. From these years, the very high frequency (VHF) band was mainly used for radiotelephony services between pilots and controllers. It has been further augmented with Satellite Communication (SATCOM) since the early 1990s. Hence, voice communications are possible even in oceanic areas where direct communications with VHF ground stations cannot be deployed regarding their range.

Nevertheless, considering the increasing number of aircraft in airspace, the lack of resources makes it necessary to foresee new solutions in order to avoid congestion. Data link (or digital data link) came up as the promising solution. Data link indeed provides the ability to transmit short and relatively simple digital messages between aircraft and ground stations via communication systems. In July 1978, the engineering department at Aeronautical Radio Incorporated (ARINC) introduced a first data link means known as Aircraft Communications Addressing and Reporting System (ACARS). And during the 1980s, air traffic control (ATC) authorities promoted the use of ACARS between controllers and pilots to improve the safety and efficiency of air traffic management. At the beginning of the 1980s, the International Civil Aviation Organization (ICAO) created a special committee known as Future Air Navigation System (FANS), where Boeing and then Airbus developed their FANS products, known as FANS-1/A. 


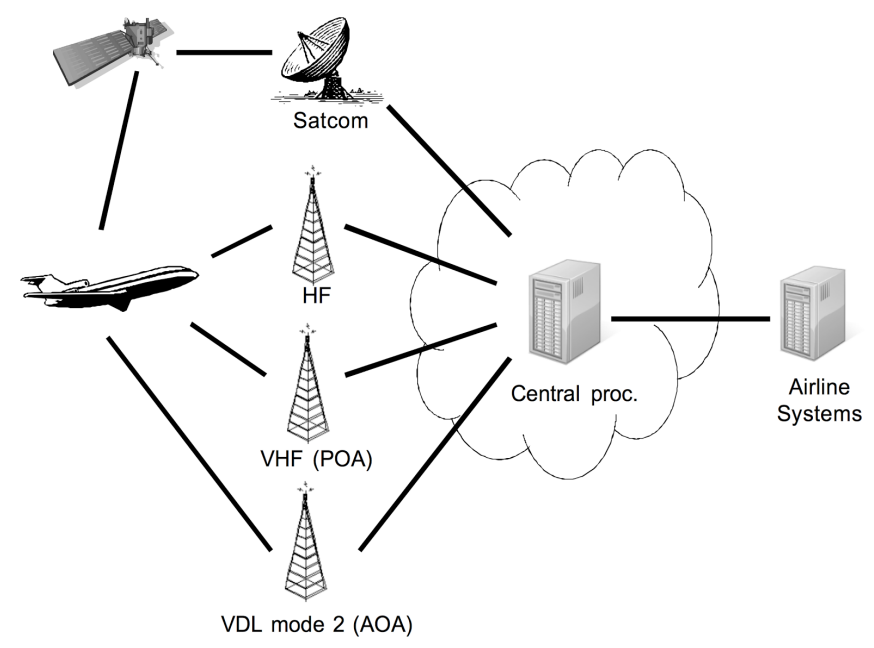

Fig. 1. Air-ground communication systems

According to ICAO, four categories of communications are defined in aeronautical telecommunications. Air Traffic Service Communications (ATSCs) and Aeronautical Operational Control (AOC) Communications that are considered as safety related, and Airline Administration Communications (AACs) and Aeronautical Passenger Communications (APCs) that group non-safety-related. Critical communications follow specific international rules defined by ICAO (for example, only some dedicated frequency band can be used) and are based on dedicated systems. These latter must meet very stringent QoS requirements mainly based on transaction time, continuity, availability and integrity. In this paper, our focus will be on critical services (ATSC and AOC).

Airbus and Boeing worked on a first set of applications using the available technologies for air-ground data communications focusing on oceanic and remote airspaces with no radar coverage and poor HF voice communications. These applications thus addressed communication and surveillance needs for these airspaces.

In parallel of the deployment of FANS-1/A, ICAO working groups continued to develop standards for a new aeronautical dedicated network and a set of applications: ATN (Aeronautical Telecommunication Network). In addition to ground-ground applications, ATN also defines air-ground data link applications, similar to those of FANS 1/A with some modifications and enhancements released in FANS-2/B. Besides, ATN defines a global internetwork architecture. As such, it relies on different "real" subnetworks, allowing interconnections between ATN routers. ATN defines a stack of OSI standardized protocols from the network layer up to the application layer. ICAO working groups also developed standards for the underlying air-ground subnetworks: in the VHF and HF bands, and also through satellite. More specifically, several technical choices or protocol stacks were proposed in the VHF band and are called VDL (VHF data link) mode 2 to 4 . Thus, considering aeronautical data link communications, in each successive generation, we found a set of application services (FANS), which 
uses an upper layer architecture based on lower layer architectures and radio systems (HF, VHF, SATCOM, etc.).

\section{2 $\quad$ Future trends}

The aviation industry has identified the need to develop new data communication protocols and services to meet the safety and performance requirements of aviation for the year 2020 and beyond. Besides, the aviation industry desires a modern, off-theshelf, efficient, and robust network infrastructure common to both ATS and AOC safety services. Commercial IP has been identified to be the successor in the long term of the ATN/OSI network infrastructure. It will be referred as Aeronautical Telecommunication Network/Internet Protocol Suite (ATN/IPS), based on IPv6. Indeed, the Internet Protocol IPv6 is a widespread telecommunications standard in the industry that is maintained and extended by the IETF. It will favor the interconnection between aeronautical domains that have already begun to migrate to IPv6, like for instance ground networks. Also, IPv6 should support a world-wide deployment with enough address space. It will be implemented on both aircraft and ground infrastructure. It is expected to use multiple line-of-sight and beyond-line-of-sight subnetworks such as Inmarsat SwiftBroadband, Iridium Certus, AeroMACS, future SATCOM and L Band Digital Aviation Communication System (LDACS) systems, and VDL Mode 2.

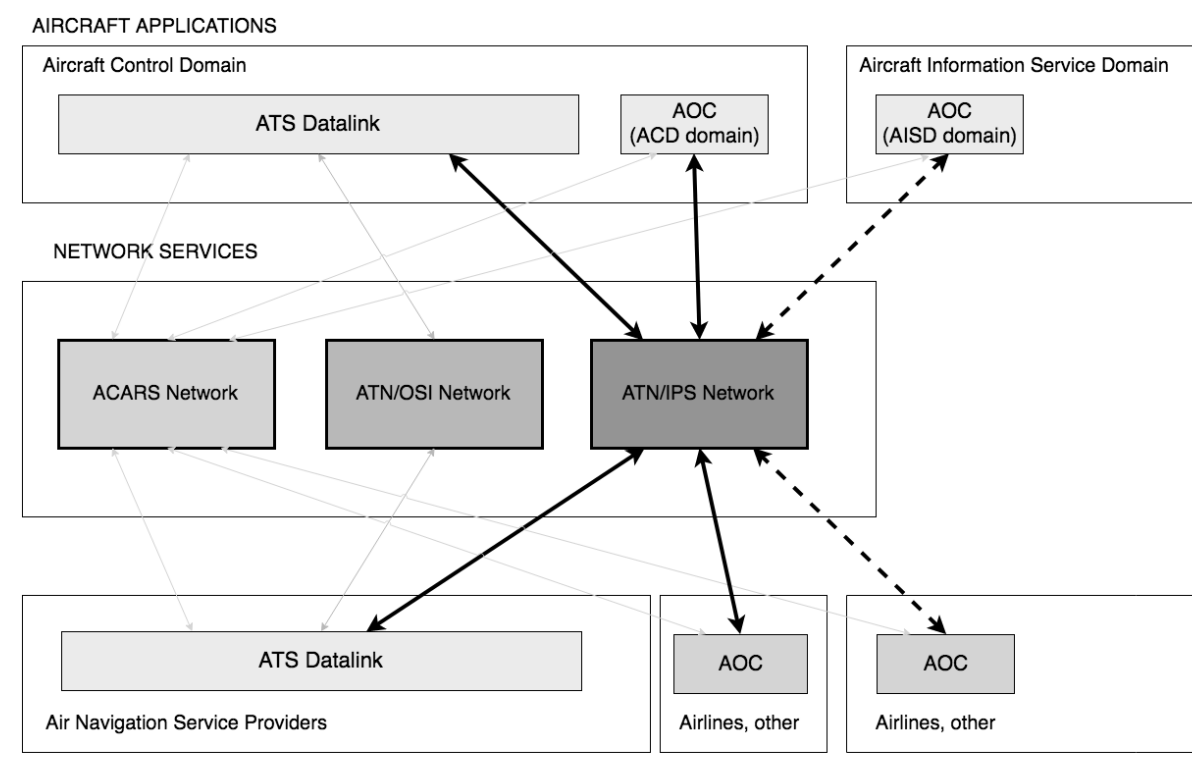

GROUND APPLICATIONS

Fig. 2. ATN/IPS implementation (from [4])

ATN/IPS should benefit for all the actors. Greater communications are expected for airlines compared to ACARS and ATN/OSI, while avionic suppliers and airframe manufacturers will be capable to provide more bandwidth and capabilities by using future data link technologies. The Fig. 2 shows how the ATN/IPS network should be used in the future. 


\section{IP Mobility}

The need of being always connected to the Internet has significantly arisen since the last decade. Internet mobility has thus become an attractive research area over the years. Several solutions have been proposed to provide a seamless communication for an end user while on the move and changing his access network to the Internet. The process of switching onto different networks is called handover. The handover process is either horizontal or vertical, depending on whether it switches to the same network technology or a different one. A handover is efficient if the user does not perceive any interruption. The main difficulty is related to the fact that application sessions are identified with an IP address, so changing network access and thus IP address may result in application sessions being broken. Therefore, a handover mechanism that keeps the IP address will be transparent to the application layer.

\subsection{Mobility main principles and MIPv6 protocol}

Several solutions based on different layers can deal with the mobility in IP networks [1]. The more adapted solution to the aeronautical environment according to [1] is based on the network layer. Indeed, providing a solution above the network layer is not suited for a deployment in an aeronautical environment as it should be implemented on all the existing end systems. Besides, considering the aircraft as a mobile network, it makes more sense to manage the mobility issue at the network layer to avoid doing it for each running application. More details on other possibilities to handle the mobility in IP networks are presented in [1].

In the context of IPv6, the IETF has developed an extension to deal with the mobile nodes (MNs) in the network layer which is Mobile IPv6 (MIPv6) [2]. It provides an efficient and scalable mechanism to handle the node mobility in IP networks. Indeed, it allows mobile nodes to move and change their point of attachment without changing their IP address. Therefore, this mechanism is transparent to the upper layers and allows sessions continuity during a handover.

To do so, MIPv6 uses 2 IP addresses for each mobile node: a Home Address (HoA) and a Care-of-Address (CoA). The first one identifies the node in its home network and the second one allows to locate the node when it moves to a foreign network. The association of the 2 addresses is realized by an entity in the home network called the Home Agent (HA). Each time a MN attaches to a foreign network, it performs a binding association between its new CoA and its HoA by sending a Binding Update message to its Home Agent, which replies with a Binding Acknowledgement message. The HA then creates a bi-directional tunnel to forward traffic to the new location of the $\mathrm{MN}$. A correspondent node $(\mathrm{CN})$ communicates with the mobile node by using its HoA. Therefore, when a MN performs a handover to a new IP network, it remains transparent for its CNs.

This mechanism allows session continuity as all packets coming from CNs are captured by the Home Agent and then forwarded to MNs. However, it introduces a triangular routing because packets sent by the mobile node are forwarded by standard IP routes. To solve this issue, MIPv6 allows MNs to perform route optimization (RO) with their CNs. Route Optimization is carried out after a binding association. MN 
creates a secure bi-directional tunnel with its CNs so that packets are exchanged directly through this tunnel.

\subsection{MIPv6 enhancements}

Whereas MIPv6 can provide session continuity during a handover phase, its mechanism is not well adapted in specific environments such as in aviation, or for some specific applications which require very constraint requirements. For instance, very long links have a direct impact on the handover delay and thus introduce a long period during which the mobile node is unreachable. In the following, some enhancements to MIPv6 are described in order to mitigate these issues.

HMIPv6 (Hierarchical MIPv6). HMIPv6 is introduced in [5] as an extension to MIPv6 and IPv6 Neighbor Discovery protocol (NDP). It helps reducing the signaling traffic during the handover phase for local mobility case thanks to a new entity called the mobility anchor point (MAP). It works as a local HA for a MN. HMIPv6 separates the global network into different MAP domains, each one controlled by one or several MAPs (see Fig. 3). A MAP domain is different from a network domain. HMIPv6 introduces 2 addresses to manage the local mobility of a node: the regional care-of-address $(\mathrm{RCOA})$ and the local care-of-address (LCoA). The first one (RCoA) is used to realize the binding with the $\mathrm{HA}$ and the $\mathrm{CN}$, if the route optimization procedure is triggered. MN obtains a new RCoA whenever it moves to another MAP domain and attaches to a new MAP. Meanwhile, the second one (LCoA) is used as a binding with the $\mathrm{RCoA}$ in the MAP domain. This process allows the MAP to forward packets destined to a $\mathrm{MN}$ in its current location through the tunnel created between the RCoA and the LCoA. Besides, HMIPv6 makes the local mobility in a MAP domain transparent to the $\mathrm{HA}$ and the $\mathrm{CN}$ as the RCoA is not updated.

In consequence, HMIPv6 is more suitable for local mobility but presents some drawbacks in dealing with global mobility because MNs need to get 2 different IP addresses instead of only 1 with MIPv6.

FMIPv6 (Fast handovers for MIPv6). FMIPv6, described in [6] [7] as a further enhancement to MIPv6, is a protocol that helps reducing the handover latency and the packet loss during a handover in MIPv6. FMIPv6 makes it possible by introducing a fast binding between MN's previous access router (PAR) and MN's next access router (NAR). This tunnel is created before the binding with the HA. To do so, during the discovery phase, all the access routers must share information about their network prefix so that MN can configure the new CoA accordingly. With this tunnel, data may be tunneled to the new access router even during the handover phase.

By introducing some complexity at the network level, FMIPv6 is more effective than MIPv6 during a handover as it allows to mitigate the negative effects such as handover latency and packet loss. 


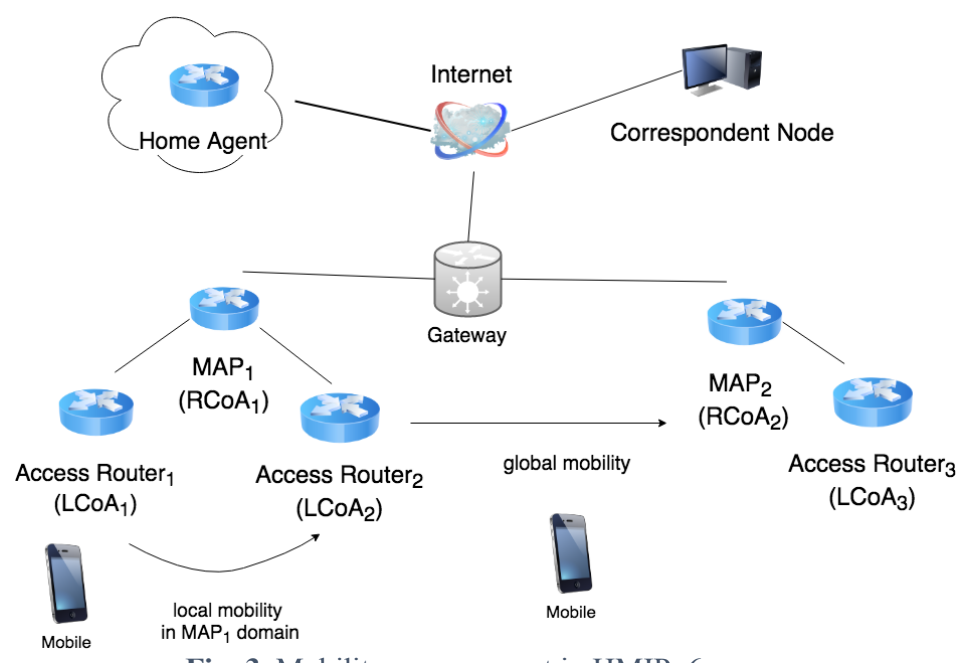

Fig. 3. Mobility management in HMIPv6

PMIPv6 (Proxy MIPv6). PMIPv6 [8] is a network-based mobility management. Unlike the previous protocols which are host-based approaches, the node mobility is handled by access routers in the network. Its main interest is to reduced drastically the amount of signaling for the mobile node as it is no more involved in the process. Like HMIPv6, PMIPv6 provides mobility support within a domain called a PMIP domain. As long as the MN moves within the domain, the network has to track the location of MN, which keeps its IP address.

PMIPv6 introduces 2 new entities to manage the node mobility: the mobile access gateway (MAG) and the local mobility anchor (LMA). The MAG realizes the mobility-related signaling on behalf of the $\mathrm{MN}$ using its access links. It is responsible for detecting the movement of the nodes in the domain and for executing binding registration with the corresponding LMA. The LMA manages the routes for all mobile nodes in the domain. More information about the whole process are developed in [7] [8] [9].

In consequence, whereas similar to HMIPv6, PMIPv6 seems to be more appealing because it does not involve the $\mathrm{MN}$ in the process, and hence helps to reduce signaling traffic seen by the MN.

\section{$4 \quad$ MIPv6 performances assessment in an aeronautical context}

During a flight, an aircraft will be covered successively and/or simultaneously by several air-ground subnetworks belonging to different administrative domains (ANSPs and ACSPs) depending on its location.

In the current aviation network, an aircraft has a unique identifier (its ICAO address) by which it is reachable at any times. But moving towards different IP networks, the aircraft must obtain an IP address corresponding to the air-ground subnetwork in use in order to maintain its reachability. Consequently, an IP mobility mechanism is required to maintain the ongoing sessions with the aircraft whenever it changes 
its point of attachment to the air-ground network. Session continuity must be carefully taken into consideration during the process. Indeed, it should not break the ongoing sessions while performing the handover. However, a session in a TCP/IP stack is broken as soon as the IP address is changed.

As a first step of our research work, we decided to perform an evaluation of MIPv6 in a ATN/IPS representative network that will serve as a reference for our future proposal. Indeed, the aeronautical network infrastructure is not like a common ground network equipped with $4 \mathrm{G}$, due to its limited air-ground link capacity coupled with very demanding requirements of ATS applications running on top of that. Therefore, metrics such as packet loss, handover delay, and signaling traffic must be carefully investigated.

A previous work has already been carried out in [10] to evaluate MIPv6 for ATN/IPS. It highlights the fact that handover delay can be very long in particular scenarios.

Our simulation environment is based on Omnet $++[11]$, a discrete event simulator adapted for wireless communication and its IPv6 framework INET. Omnet++ is based on a modular architecture, thus make it simple to use existing modules and to develop our own ones. The INET framework provides a xMIPv6 module which simulates the standard MIPv6 protocol described in [2]. For now, the xMIPv6 module was tested in a simple scenario.

\subsection{Simulation scenario, parameters and hypothesis}

Links modeling and MIPv6 module. As we do not intend to perform an analysis on link technologies, we have implemented a simple wireless NIC (Network Interface Controller) on each node and router. The wireless NIC uses the well-known CSMA protocol at the MAC layer as in some existing air-ground systems like VDL mode 2, and an ideal physical channel free of errors (bit error, collision...). Hence, base stations have been omitted as the mobile nodes communicate directly with routers equipped with a wireless NIC. In doing so, it allows us to focus only on network layer mechanisms. Besides, movement detection at the network layer is performed when MNs receive an Router Advertisement (RA) announcing a new network prefix. The RFC 6275 recommends to set the RA periodicity between $30 \mathrm{~ms}$ and $70 \mathrm{~ms}$. This value seems very low in our context. As it is a source of signaling traffic, the value has been increased up to $300 \mathrm{~ms}$. A further analysis on the impact of this parameter will be investigated. Besides, route optimization has been removed from the MIPv6 module because ATS traffic may not be forwarded by other non-certified providers.

Ground network. For a first step in our simulation, a simple handover between 2 access routers has been tested out, with one representing the HA for the mobile node, as shown in Fig. 4. It's a realistic scenario if we consider a continental scenario like in Europe where connectivity to the ground network is provided by two Aeronautical Communication Service Providers (ACSPs): Rockwell Collins IMS (formerly ARINC) and SITA. Meanwhile, delay between those routers are modeled based on the guaranteed maximum delay between the 2 ACSPs [10]. For ATS services, the communication end point is an ATSU (Air Traffic Services Unit), which depends on the location of the 
aircraft. For now, the application layer integrates a PING application which sends ICMP messages periodically.

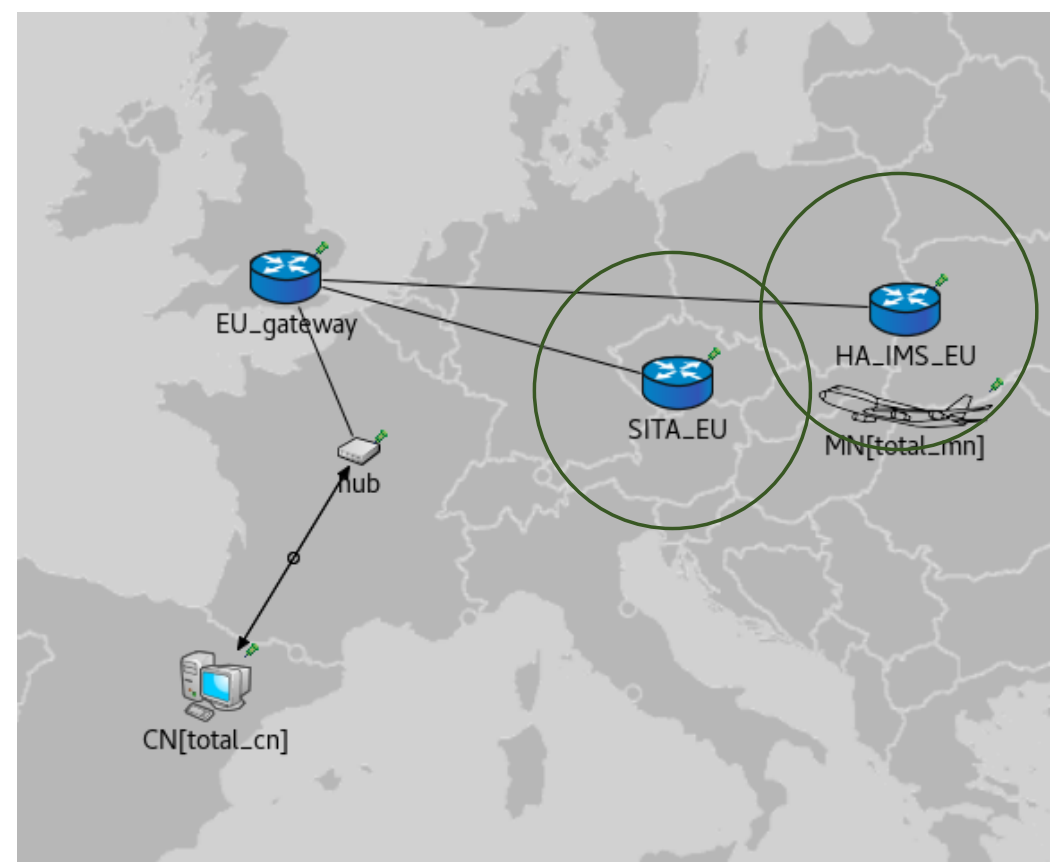

Fig. 4. Simulation scenario

Scenario. We consider one aircraft during its en-route phase at a cruise speed of 220 mps. It is first covered by IMS ground stations which is its HA and flies to a region covered only by SITA ones. The overlapping area, corresponding to the area where the aircraft has an access to both IMS and SITA, is about $50 \mathrm{~km}$ long.

As the IdealRadioMedium module is used to simulate the wireless medium, there is no link mechanism to associate the aircraft with only one access router on a dedicated channel. Therefore, when the aircraft goes through the overlapping area, it will receive both network prefixes of IMS and SITA. The MIPv6 module running on the aircraft will thus try to attach to both access networks successively as it will continue to receive RAs even after performing a binding with its new $\mathrm{CoA}$. This phenomenon is known as the ping pong effect. A handover manager is therefore necessary to avoid this effect.

Handover manager at L2. We thus decide to integrate a handover manager at the layer 2. It is an intermediate layer between the link layer and the network layer. Its role is to filter incoming packets at the network layer of aircraft. Its filtering is based on a predefined priority list of ground stations. When it receives a beacon from a ground station, it checks whether this ground station has a greater priority than the current ground station in use. While this is a static and simple mechanism, it can be easily adapted in the future to a more dynamical decision process by modifying the priority list accordingly. 
To implement our handover algorithm, layer 2 beacons using MAC addresses have been added. They are sent every $300 \mathrm{~ms}$ by each access router with their respective MAC address and their ground station ID. RA messages of the MIPv6 module cannot be used for this purpose because our handover manager is run under the network layer, thus it does not manipulate IP addresses. Another more complex solution has been standardized in IEEE 802.21 [12], referred as Media Independent Handover (MIH) to manage efficiently the handover between different wired and wireless networks. Unfortunately, it is not yet developed for Omnet++ and is not our primary concern, that is why another approach has been taken.

\subsection{Results and analysis}

In order to validate our proposal, several simulations have been run using MIPv6 mechanism on one side, and MIPv6 coupled with our handover manager on another side.

Fig. 5 shows packets received by the aircraft at the link layer. By inspecting the size of packets, we deduce their type, thus helping us to determine the impact of our system on MIPv6.
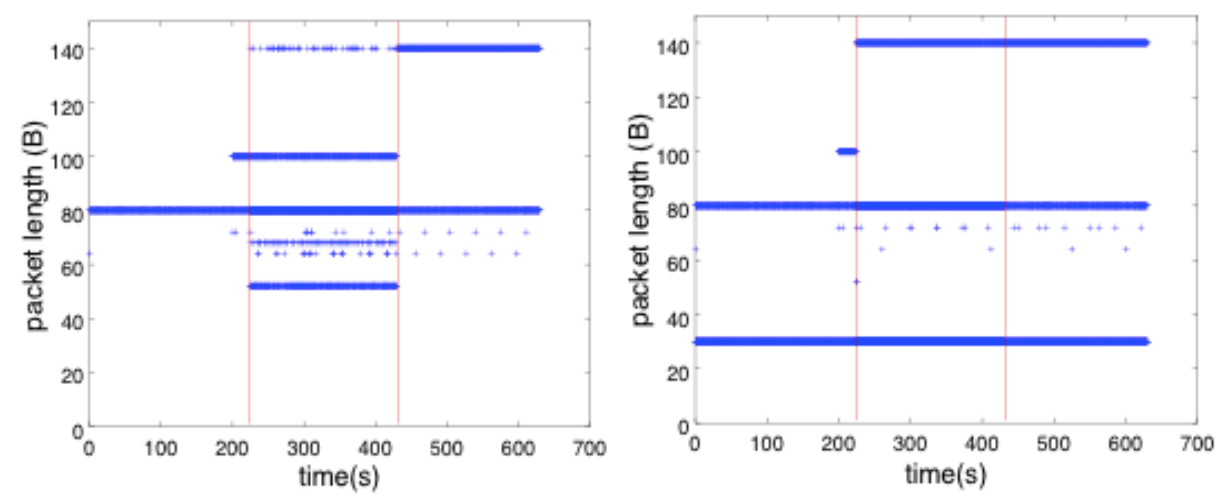

Fig. 5. Packets received by the aircraft in an ideal case on the left, and with our manager on the right

Before a handover occurs, the aircraft only sees its HA, thus all the PING packets it receives come from the HA. Fig.5 illustrates the same behavior in both cases (for $t<224 \mathrm{~s}$ ), therefore our handover mechanism does not impact this phase. It only induced an additional signaling traffic coming from router beacons.

When the aircraft enters the overlapping area, it will have the choice between the 2 access routers. In the ideal case, left figure in Fig. 5, between 220s and 430s, the aircraft receives some PING packets from the SITA ground station, highlighting the ping pong effect. Those packets are longer ( 140 bytes instead of 100 bytes) than those sending by the HA because of the additional header corresponding to the tunneling mechanism. Whereas using our handover mechanism, right figure in Fig. 5, the aircraft 
only receives PING packets of size 140 bytes, thus coming from the SITA station. Indeed, as soon as the aircraft detects the SITA ground station, it creates the binding and maintains it because SITA ground station gets a greater priority, as expected.

More interesting is the number of packet loss at the application layer in both case shown in Fig. 6. With our handover manager, although there are still some lost packets, the number of loss is significantly reduced when the aircraft is covered by the 2 access networks (between 220 s and 430s).

These first results validate our handover mechanism proposal. While being very elementary, it allows us to handle IP mobility effectively with MIPv6. Nevertheless, the long handover delay mentioned in [10] and the number of messages exchanged during the handover by the aircraft may prevent from meeting the stringent requirements for performance of the ATS traffic. From the IP solutions introduced earlier, PMIPv6 seems to be a good candidate to overcome these issues as the signaling traffic on the air-ground link will be significantly lower.

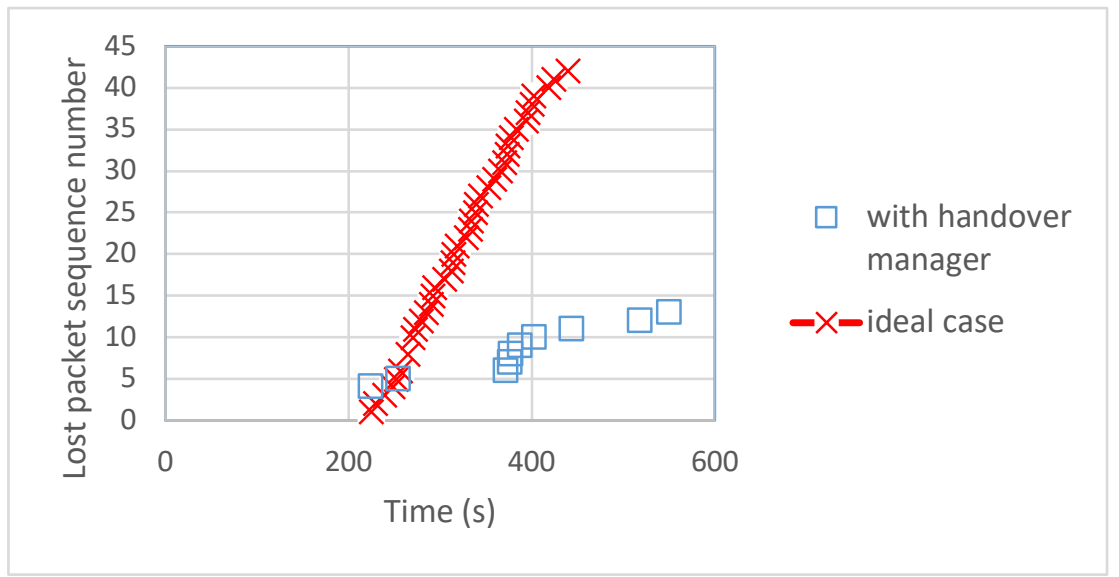

Fig. 6. Packet loss in both scenarios

\section{$5 \quad$ Conclusions and further work}

In this paper, we have presented the aeronautical communication environment and its evolution towards IP-based communications. We then focus our attention on the mobility issue and the solutions to manage it at the network layer. As a first step, we have started to assess the suitability of MIPv6 for an aeronautical environment with a representative simulation model in Omnet++. The first results have shown that MIPv6 does not provide any handover mechanism, that is why we decided to develop a layer 2-based handover manager. Without adding any messages during the handover process, our handover manager avoids MIPv6 protocol to be affected by the ping pong effect during a handover.

Further works have already been identified. They can be separated into 2 axes. Firstly, enhancements to our model are possible through (a) a more realistic application layer simulating an ATS traffic, (b) a modeling of other air-ground access networks like SATCOM that will helps us to test transatlantic flight scenarios representative of 
vertical handover scenarios and (c) generating exogenous traffic by integrating several flights in our scenario or by adding external traffic in access routers to measure their impact on the handover procedure in MIPv6. Secondly, a contribution to IP mobility in Omnet++ has been pinpointed. Indeed, in our aeronautical context, MIPv6 seems to present some drawbacks in terms of signaling and handover delay. PMIPv6 may offer better performances regarding these criteria, but it is not yet implement in any Omnet++ framework. Nevertheless, none of these solutions has been designed to be implemented on a very specific environment such as civil aviation, therefore we may adapt PMIPv6 to our scenarios.

\section{References}

1. C. Bauer, M. Zitterbart: «A Survey of Protocols to Support IP Mobility in Aeronautical Communications». In: IEEE Communications Surveys and Tutorials, vol. 13, pp. 642-657, fourth quarter 2011.

2. D. Johnson, J. Arkko: «Mobility Support in IPv6, RFC 6275». C.Perkings, July 2011.

3. F. Y., C. Bauer, C. Wietfield: «An accurate and extensible mobile IPv6 (xMIPv6) simulation model for Omnet++». In: Omnet++2008: Proceeding of the 1st International workshop on OMNET++, 2008.

4. AEEC: «Internet Protocol Suite (IPS) for Aaeronautical Safety Services Roadmap document». SAE ITC, Bowie, Maryland, 2017.

5. H. Soliman: «Hierarchichal Mobile IPv6 (HMIPv6) Mobility Management, RFC 5380». 2008.

6. R. Koodli: «Mobile IPv6 Fast Handovers, RFC 5568». 2009.

7. D. Li, Z. Li: «Optimization and Enhancement of MIPv6 in ATN». In: Aeronautical telecommunications Network : Advances, Challenges, and Modeling. Boca Raton, CRC Press, 2015.

8. S. Gundavelli, K. Leung, V. Devarapalli, K. Chowdhurry: «Proxy Mobile IPv6, RFC5213». 2008.

9. I. Soto, C. J. Bernados, al.: «PMIPv6: A Network-Based Localized Mobility Management Solution». In: The Internet Protocol Journal, vol. 13, pp. 2-16, 2010.

10. C. Bauer, S. Ayaz: «A Thorough Investigation of Mobile IPv6 for the Aeronautical Environment». In:IEEE Vehicular Technology Conference-Fall Proceedings, pp. 1-5, 2008.

11. A. Vargas, al.: «The Omnet++ discrete event simulator,» 2005. https://www.omnetpp.org/ .

12. A. De La Oliva, A. Banchs, al.: «An overview of IEEE 802.21: media-independent handover services». In: IEEE Wireless communications, vol. 15, 2008. 
